\title{
POUR UNE CULTURE RÉTICULAIRE : LA PERTINENCE DU PARADIGME MÉDITERRANÉEN
}

\author{
Marie-Joseph Bertini ${ }^{1}$
}

"Cette mer -écrit Hegel à propos de la Méditerranée- est une grande puissance naturelle dont l'influence est décisive : nous ne pourrions pas imaginer le cours de l'Histoire si nous ne tenions en elle un élément central de conjonction"2. L'objet de cet article s'articule autour de deux hypothèses : il est possible et utile de dégager un véritable paradigme méditerranéen qui oriente et éclaire, sous conditions, la constitution de certains types de phénomènes culturels.

C'est ce paradigme, au sein duquel s'originent les conditions de possibilité des premières cultures, qui nous semble à nouveau à l'œuvre aujourd'hui dans la définition et la mise en place des grands réseaux d'information et de communication : ce qui constitue notre seconde hypothèse de travail. Peu patente de prime abord, la validation de cette dernière hypothèse favorise, comme nous le verrons, l'intelligibilité des nouveaux modes de production et de diffusion des cultures en réseaux virtuels.

1 Maître de conférences à l'Université de Nice-Sophia Antipolis. Chercheur au CREA (Centre de recherches en anthropologie, communication et création) Institut méditerranéen IMAGE.

2 G. W. F. Hegel, La Ruse de la Raison, Paris, Plon, 1965, p. 244.

Recherches en communication, $\mathrm{n}^{\circ}$ 9, (1998). 


\section{Qu'est-ce que la Méditerranée?}

Appréhender l'objet Méditerranée dans son historicité, c'est se confronter à un mélange complexe de déterminismes naturels, de vicissitudes historiques, et de particularismes éthniques desquels émerge ce prodige hautement improbable : le Monde Méditerranéen. Car c'est bien d'un univers en soi qu'il s'agit, c'est-à-dire un ensemble cohérent constituant un système organisé. Ainsi l'extrême diversité de sa géographie, la prodigalité de ses cultures, et la bigarrure des peuples qui la composent, ne doivent-ils pas nous masquer l'essentiel : l'exceptionnelle faculté de créer -par delà les différences et les conflits violents qui les opposèrent ${ }^{1}-$ les conditions d'une unité singulière fondée sur un métissage plusieurs fois millénaire. "A ce monde épais, composite et mal délimité... on ne peut reconnaître d'autre unité que celle d'être une rencontre des hommes, un alliage d'histoire" écrit Fernand Braudel ${ }^{2}$.

A la question $Q u ' e s t-c e$ que la Méditerranée? on peut donc répondre: moins une juxtaposition de territoires qu'un espace commun $^{3}$, moins une histoire qu'une catégorie trans-historique, moins une profusion de cultures qu'une aptitude à les transcender toutes, en une hétérogénéité vivante et combinatoire.

L'idée de cette altérité qui communie dans une unité, de ce "très vieux carrefour" dont Hermès est le dieu tutélaire, s'impose à nous. En ce sens la Méditerranée se définit avant tout comme une dynamique de circulation des personnes et des biens, à travers les routes de terre et les routes de mer.

"La Méditerranée n'a donc d'unité que par le mouvement des individus, les liaisons qu'il implique, les routes qui le conduisent" ${ }^{4}$. La Méditerranée, c'est cet "espace-mouvement" dont émergent les

1 La Méditerranée, c'est aussi l'histoire des "identités tenaces, des refus héréditaires, des guerres meurtrières, des passions destructrices": M. ARKOUN, "Une autre histoire de la pensée en Méditerranée", in La question méditerranéenne, Paris, Éd. du Seuil, 1988, p. 80.

$2 \mathrm{~F}$. BRAUdel, La Méditerranée et le monde contemporain à l'époque de Philippe II, tome 1, La part du milieu, Paris, Armand Colin, 1990, p. 279.

3 La notion d'espace contient l'idée d" "écarts", de "différences" adjacentes qui sont en relation d'"extériorité" les unes par rapport aux autres. Elles sont en outre différemment placées : certaines au-dessus, d'autres au-dessous, d'autres encore entre : cf. P. Bourdieu, Raisons pratiques. Sur la théorie de l'action, Paris, Éd. du Seuil, 1994.

4 F. BRAUDEL, op. cit., p. 338. 
Figures primordiales du marin et du trafiquant, indispensables médiateurs, agents de pénétration des cultures des pays de l'intérieur de la Méditerranée orientale vers les côtes, passeurs anonymes amplifiant sans cesse la démesure du brassage méditerranéen. Brillants représentants des cultures côtières de Syrie et de Palestine, les Phéniciens effectueront des échanges incessants entre Mésopotamie, Anatolie, Egéide et Egypte. Des siècles plus tard, Théophile Gautier émerveillé, décrira dans son Voyage à Constantinople le mélange bigarré de toutes les cultures peuplant de leur vie bruyante ports et escales : Grecs, Arméniens, Albanais, Levantins, Juifs, Turcs, Italiens... Ce syncrétisme, qui suppose la diversité des éléments qui le composent, innerve l'immense réseau des relations régulières et fortuites, distribuant biens matériels et symboliques, quand se croisent dans un mouvement ininterrompu, les armes et les croyances, les bijoux et les langues, les techniques anciennes et les arts nouveaux.

Pour toutes ces raisons, la Méditerranée constitue une entité unique dont aucun autre espace géographique n'a pu ou su -de l'aveu même des historiens et des philosophes- dégager les propriétés singulières. Hegel encore: "Ainsi la Méditerranée est le cœur du Vieux-Monde, elle le conditionne et l'anime"].

\section{Réticulations méditerranéennes : pèlerins d'hier et d'aujourd'hui}

Dès les origines donc, la Méditerranée ne se laisse penser que sous la catégorie du réseau, cet ensemble de connexions multiples et constantes, possédant une unité invisible. Puissance circulatoire qui est la métaphore de la vie même, comme le montre Saint-Simon dans son Introduction aux travaux scientifiques $d u X^{X} X^{e}$ siècle ${ }^{2}$, soulignant l'homologie des catégories de réseau et d'organisme, dont le corps humain, cathédrale de rhizomes mobiles et enchevêtrés, lui fournit la riche métaphore. L'organisme et sa cohérence interne assurée par les flux ininterrompus, deviennent les référents naturels d'une théorie de l'organisation à laquelle la révolution industrielle donnera ses accents

1 Ibid., p. 243

2 In Euvres, tome VI, Paris, Anthropos, 1966, p. 66. Cf. "Anciennes nations, nouveaux réseaux", Les Cahiers de médiologie, Paris, Gallimard, $\mathrm{n}^{\circ}$ 3, 1997. 
modernes, rompant ainsi avec l'imaginaire mécaniciste hérité du XVII siècle.

La circularité est donc l'essence de la Méditerranée, et l'équilibre de ses systèmes naturels et artificiels en dépend : tel est le sens de l'archétype du Pèlerin, traceur des liens virtuels entre l'ici et l'ailleurs, le sensible et l'intelligible, le présent du monde et son souvenir à jamais perpétué. Terre de pèlerinage, de Délos à Compostelle, en passant par Delphes et Jérusalem, la Méditerranée est traversée de tous côtés par ces "peregrini", littéralement ces "voyageurs étrangers" qui suivent le long cortège de l'exode et des prophètes errants dans le désert. En ces temps lointains, le Pèlerin fait signe de l'humaine condition soumise aux lois de la traversée des apparences : passants égarés, nous ne sommes que de passage, nous rappellent-ils, en route vers ces lieux saints qui forment les points d'accès à la Jérusalem Céleste. Pensons ici à une autre Figure symboliquement forte : celle du Juif qui originairement exprime cette altérité radicale qui divise et rassemble, cette ubiquité de celui qui nulle part ne se laisse enraciner. Pur flux, opérateur d'échanges multiples, nomade se jouant déjà des frontières et des empires, il est trop en avance sur son temps pour ne pas incarner cet autre archétype, cher à René Girard : le boucémissaire. Soulignant cette précocité douloureuse, Fernand Braudel usera d'une formule audacieuse : "Il est", écrit-il, "une modernité qui a trop d'avance à l'allumage"1.

Comme le Juif errant, le Pèlerin et le Croisé nous parlent à mivoix d'un arrière-monde, visible dans l'ombre projetée des sanctuaires qui assemblent et réparent les mémoires déchirées par l'oubli. C'est ce contrat moral, et donc social, que Michel Serres célèbre dans son Atlas des mondes nouveaux, en insistant sur ce devoir de "couture" coincé entre l'utopie du partage et la tragédie du négatif ${ }^{2}$.

L'ensemble des éléments qui précèdent permet donc de dégager l'idée d'un modèle spécifiquement méditerranéen, désignant la convergence des facultés de reliance ${ }^{3}$, de circularité dynamique, de contre-partie stratégique, de recomposition des relations symboliques, et de métissage. Par ce terme, il faut entendre à la fois l'hybridation, ce croisement fécond, mais aussi l'influence de la Mètis des Grecs,

1 F. BRAUDEL, op. cit., tome 2, Destins collectifs et mouvements d'ensemble, p. 566.

2 M. SERrEs, Atlas, Paris, Flammarion, 1996, p. 196.

3 Cf. M. Bolle DE BAL, La tentation communautaire. Les paradoxes de la reliance et de la contre-culture, Bruxelles, Université de Bruxelles, 1985. 
déesse gouvernant la pure ingéniosité, la tactique habile qui combine ruses et expédients, et dont Ulysse demeure le type emblématique ${ }^{1}$.

Creuset des identités disséminées et variées, ce modèle méditerranéen permet de formuler les conditions d'un être-ensemble original, triomphant des exigences multiples et contradictoires des éléments qui le constituent.

Il paraît donc utile à présent de systématiser son champ d'application en montrant combien la pertinence de ce modèle est requise dans l'analyse de la nature et des enjeux des nouvelles opérations culturelles en réseaux.

\section{La dynamique des opérations culturelles en réseaux}

En mettant à la libre disposition de tous, les contenus et les formes les plus hétéroclites : bibliothèques, œuvres d'art, recettes de cuisine, informations scientifiques, jeux, idées, humeurs... ce que Walter Benjamin appellerait "le concret le plus extrême", les nouvelles technologies d'information et de communication que sont les réseaux électroniques ( Internet, BBS, Dante, Europanet, Usenet, WEB ...) constituent le moteur d'une nouvelle économie culturelle. En procédant à une redistribution des savoirs et des savoir-faire, mais aussi en restituant aux individus des outils d'échange d'émotions partagées, les réseaux virtuels tissent la trame de nouvelles communautés. Analyser en effet la problématique des réseaux sous l'angle communautaire, est un bon moyen d'éviter les effets rhétoriques de la trop fameuse "dissolution du lien social".

Max Weber mettra en valeur l'importance, à travers l'Histoire, de ces "communautés émotionnelles" (Gemeinde) surgies des religions et des cultes. Leur rôle consistait à socialiser les individus qui y prenaient part, en les soudant par un "sentiment commun spécifique" exprimant la naissance d'une "conscience tribale" fondement d'une nouvelle communauté politique ${ }^{2}$.

L'étude approfondie des communautés issues des réseaux révèle un certain nombre de constantes. Ce phénomène déborde largement le cadre des États-Unis puisque ces rassemblements s'effectuent dans

1 Cf. M. Detienne et J.-P. Vernant, Les ruses de l'intelligence : la Mètis des Grecs, Paris, Flammarion, 1989.

2 M. WEBER, Économie et Société, tome 2, L'organisation et les puissances de la société dans leur rapport avec l'économie, Paris, Agora Pocket, 1995, p. 204-211. 
plusieurs pays. En effet The WELL, GeoCities, Electric Minds aux USA ne doivent pas nous faire oublier TWICKS à Tokyo, Chaos Computer Club à Hambourg, RTC Alternatif en France, Hacktick aux Pays-Bas, Club Dog à Londres, Ciudad Futura en Espagne parmi d'autres exemples. Il s'en dégage l'évidence d'un puissant sentiment d'appartenance communautaire, de l'aveu même de ses protagonistes ${ }^{1}$. L'hebdomadaire Business Week vient de publier un sondage réalisé par Harris Poll, montrant que $57 \%$ des internautes préfèrent utiliser toujours les mêmes sites plutôt que de surfer sur des sites inconnus, et que $89 \%$ des usagers du courrier électronique déclarent se sentir membres d'une véritable "communauté" que $35 \%$ d'entre eux assimilent à un "groupe social"2.

Comment ne pas souligner le fait que les modalités d'élaboration de ces collectifs renverse l'ordre ancien qui subsumait la géographie mentale d'une culture à la géographie physique d'un territoire? Dans le cas qui nous occupe au contraire, le lien précède le lieu en lui conférant une réalité et une raison d'être. Ni u-topiques (lieux chimériques) ni a-topiques (non lieux), les regroupements virtuels engendrent des lieux autres qui n'appartiennent pas à l'ordre symbolique classique de l'espace. Non plus être-là mais Horla (hors-là) comme déjà s'en effrayait Maupassant, l'humanité s'ab-strait davantage encore, projetée hors d'elle-même -tels Adam et Ėve chassés de l'Éden-par son langage, par ses représentations et par ses techniques ${ }^{3}$.

"La véritable valeur d'un réseau réside moins dans l'information qu'il transporte que dans la communauté qu'il forme" plaide Nicholas Négroponte 4 . C'est pourquoi cybersocialité et cyberculture pourraient bien correspondre à une recomposition des formes de la sociabilité et de la culture, à travers l'apparition de nouveaux types de configurations collectives, qui rétroagissent sur le monde réel ${ }^{5}$.

1 M. GoldwIN, "Nine principles for making communities work", Wired, juin 1994, p. 72-74. Cf. J.-C. Guedon, La planète cyber, Paris, Gallimard, 1996. Cf. A. LEMOs, "Les communautés virtuelles", Société, Paris, Dunod, n²45, 1994, p. 253260.

2 Source : <pisani@lemonde.fr>

3 Cf. M. SerRes, op. cit., p. 189 et A. LeroI-Gourhan, Le geste et la parole, tome 1 et 2, Paris, Albin Michel, 1964 et 1965.

4 N. NEGROPONTE, L'homme numérique, Paris, Laffont,1995, p. 226.

5 La théorie des trois mondes développée par Karl Popper est éclairante à ce sujet ; selon lui, l'univers consiste dans la combinaison de trois mondes: le premier monde est celui des choses ou des objets physiques, le deuxième est celui des expériences subjectives, le troisième celui des affirmations en soi. Ce dernier, dit 
Mon hypothèse est que la grande variété de nature de ces échanges virtuels s'organise en opérations culturelles qui modifient en profondeur les modalités de production et de distribution classiques de la culture. Par opérations culturelles, j'entends -en prenant appui sur les travaux et les analyses de Michel de Certeau- ce "faire-avec" qui décrit les mouvements et les trajectoires innombrables, les ruses tactiques des pratiques ordinaires : bricolages, inventions artisanales, créations singulières cachés dans les plis de l'occasion et de la circonstance, cette poïétique ou agir stratégique des individus et des groupes, dont les articulations complexes dessinent "la liberté buissonnière" des praxis. Cette grille de lecture des phénomènes culturels induit une sorte de révolution copernicienne : ce renversement nous amène en effet à cesser de privilégier les produits culturels disponibles sur le marché des biens, pour concentrer toute notre attention sur les opérations qui en font usage. Autrement dit, il s'agit ici de se retourner, par l'intermédiaire des réseaux virtuels, vers l'abondance éparse de ces créations anonymes et "périssables" que leurs opérateurs ne thésaurisent pas. En tant que telles, que représentent ces opérations en réseaux? Elles constituent de véritables actions qui modifient le réel; elles correspondent à des appropriations symboliques profondes; elles instaurent le primat d'un relationnel ubiquitaire, configuré dans le cadre de contrats librement passés avec autrui. Consultations, solidarités émergentes, conversations à bâtons rompus, dialogues argumentés, expressions de sentiments variés, confidences échappées, poèmes d'images et de mots éphémères, esthétiques fugitives... ces opérations s'inscrivent bien de manière subreptice et labile, dans l'économie culturelle générale.

En permettant à des individus et des groupes de devenir sujet de leur histoire, en réintroduisant dans le registre culturel, la dimension praxique qui en avait été soigneusement évacuée, les réseaux télématiques ouvrent des espaces d'énonciation propres, qui échappent à la surdétermination verticale des stratifications socio-économiques, et des codes culturels dominants. Espaces que Michel de Certeau appelait de ses vœux, et dont il lisait les prémisses dans l'effervescence sociale de la fin des années soixante.

Popper, est le produit de l'esprit humain ; en ce sens il est aussi réel, voire davantage, que le monde 1 et contribue grandement à sa modification.

1 L. GIARD, "Histoire d'une recherche", Introduction à M. DE CERTEAU, L'invention du quotidien, tome 1, Arts de faire, Paris, Gallimard, 1990, p. XIV. 
S'il est entendu que l'avenir seul tranchera sur la question de savoir si les réseaux constituent une matrice des mutations sociales, ils n'en sont pas moins d'ores et déjà des espaces d'opérations agissant sur les mentalités, sur les économies et sur l'organisation politique. Par quels moyens?

En créant des communautés d'intérêts partagés, par opposition (ou par juxtaposition), aux communautés de proximité classiques. Les formes de coopération traditionnelles naissaient en effet du "voisinage" qui suppléait ainsi aux forces de la communauté domestique. Le terme voisinage désignait alors "toute communauté d'intérêt, durable ou éphémère, résultant de la proximité géographique des domiciles ou des lieux de séjours"'. Pierre angulaire de l'organisation économique et sociale avec la famille, la proximité physique régulera les échanges jusqu'à l'apparition des communications à distance : ainsi les États-unis, confédération d'États indépendants, ne deviendront-ils une véritable entité culturelle qu'à partir du développement du télégraphe et du téléphone.

\section{Les réseaux, laboratoires des identités et des socialités émergentes}

C'est la raison pour laquelle les réseaux sollicitent une redéfinition essentielle du concept d'identité et d'identité culturelle en particulier : nous voyons ici s'ébaucher une identité non plus reçue passivement par l'appartenance héréditaire à un territoire et à ses traditions, mais choisie activement, à l'intérieur d'un éventail aussi large que les bibliothèques de formes, et les avatars disponibles à l'échelle des communautés virtuelles. "On the Net, nobody knows you're a dog": cette phrase amusante, bien connue des internautes, désigne clairement les réseaux comme de véritables laboratoires de l'identité. De nouvelles identités prennent leurs sources dans des prétextes très divers. Pour Howard Rheingold, la succession des masques empruntés lors des jeux interactifs (MUDS) correspond à une grammaire élémentaire du cyberespace, dont la vertu est de faire émerger une "syntaxe du jeu sur l'identité"2. Sherry Turkle voit dans ce jeu de

1 M. WEBER, op. cit., tome 2, p. 85.

2 H. RHEINGOLD, Les communautés virtuelles, Reading (Mass.), Addison-Wesley France, 1995. 
masque plus qu'un jeu : un moyen de devenir ce que nous ne sommes pas, à travers une exploration profonde des structures de notre personnalité, immanquablement modifiée par cette immersion dans des mondes que nous investissons émotionnellement ${ }^{1}$. Complexifiée, l'identité s'enrichit d'une dialectique construction-déconstruction du réel, qui nous accule à une refonte sans précédent de nos connaissances sur la psyché humaine.

Devenues soudain trop étroites, la notion d'identité, et celle connexe de société, nécessitent d'être revisitées. "Si les sociologues voulaient comprendre quelque chose au monde qui vient, ils devraient se débarrasser du concept de société" affirme sans ambages Alain Touraine ${ }^{2}$.

Car enfin qu'est-ce qui fait désormais le socius, l'allié ? Autrement dit, qu'est-ce qui détermine les nouvelles socialités ? Les flux incessants et souples, les échanges simultanés d'informations et de données diverses, les coopérations qui inversent les valeurs du proche et du lointain. Si l'on en croit le philosophe Maurice MerleauPonty dans Signes: "l'échange n'est pas un effet de la société, c'est la société même en acte". En d'autres termes, les réseaux permettent de redécouvrir l'essence de toute société vivante : non point la clôture satisfaite mais le risque du partage, non pas la sauvegarde épuisée, mais le don, l'emprunt, le détournement tactique, dans un mouvement qui relie tout projet politique à un impératif de circulation sans obstacles, une exigence de mise à disposition des savoirs.

Projet politique mais aussi pédagogique qui assigne aux réseaux la mission antique de transmission d'une culture entendue comme "paideia", c'est-à-dire éducation permanente, résolument inchoative, que Cicéron traduisit pour le monde romain par "humanitas". Humanité tranversale, présente à l'intersection exacte de toutes les cultures, jamais retenue dans l'orbe d'une seule d'entre elles.

$1 \mathrm{~S}$. TURKLE, Life on the screen. Identity in the age of the Internet, New-York, Simon \& Schuster, 1995.

2 "Sociétés en construction", Conférence d'ouverture du Congrès suisse des sciences humaines, Berne, octobre 1995. On ne peut manquer d'établir un parallèle entre la requête d'Alain Touraine et celle de Marc Augé, recommandant l'élaboration d'une "anthropologie d'urgence" destinée à rendre intelligibles ces phénomènes contemporains qui débordent les épistémologies classiques. Cf. M. AUGÉ, Pour une anthropologie des mondes contemporains, Paris, Champs-Flammarion, Paris, 1997, p. 130 . 


\section{Vers une nouvelle économie culturelle?}

Nombreuses, dès lors, sont les tentatives d'utiliser les technologies d'information et de communication pour redynamiser le tissu de nos vieilles sociétés. Citons parmi d'autres, l'opération "Métafort d'Aubervilliers", expérience de création d'une communauté mêlant nouvelles technologies, création artistique et innovation sociale ${ }^{1}$.

Portons notre attention vers ces rassemblements provisoires et opaques, dont le rôle expérimental et novateur facilite l'émergence de formes fluides et vivantes, un instant partagées ou plus durables encore, esquissant la cartographie de ces représentations de lui-même qu'un groupe appelle culture. Cette créativité qui se développe dans une réciprocité de relations et d'échanges nous plonge de plain-pied au cœur de la dialectique culturelle: "D'un côté elle est ce qui permane ; de l'autre ce qui s'invente" écrit Michel de Certeau. Dans la polyphonie de ces flux, on distingue la voix singulière des créations en acte, celles qui échappent à la pesanteur compassée des musées, celles-là même qui se déploient au-delà des propriétés artistiques qui figent l'expérience en "œuvre" et son sujet en "créateur" au génie solitaire. Les réseaux induisent ainsi des effets de transformation culturelle et politique dont la lecture reste, pour des raisons évidentes, malaisée encore.

C'est ici que le paradigme méditerranéen dégagé plus haut, revêt toute son importance. Appliqué à la culture en réseaux, il rend intelligible ce qui, sous nos yeux, est en train de se faire. Dans la nuit des câbles et des commutations électroniques, une nouvelle histoire pourrait s'écrire, mettant au jour des identités potentielles éparses, avides de se fédérer autour des écrans lumineux.

En reliant entre eux des savoirs et des individus que le hasard seul eût pu assembler, en autorisant les contreparties les plus inattendues, en recomposant les données de l'espace et du temps, en contribuant peut-être à l'avènement de ce qui pourrait être une nouvelle économie des signes et des valeurs, les réseaux et leurs périphériques constituent un méta-niveau culturel évolutif, ou niveau supérieur d'intégration des cultures au pluriel, retrouvant par là-même la force et le bien-fondé du modèle méditerranéen : interpénétration subtile des

1 "Le temps des réseaux: plein technique, vide politique", in P. CHAmbat (sous la dir.), Communication et lien social, Paris, Cité des sciences et Descartes, 1992, p. 111-135. 
formes de vie et de présence au monde, circularité, navigation, reliance, métissage stratégique de singularités, œuvrent à la définition d'un nouvel espace commun dont l'unité naît de la force et de la pluralité des échanges, alliage d'histoires multiples, pur espacemouvement dont le centre est partout et la circonférence nulle part.

Ainsi ce paradigme méditerranéen nous aide-t-il à comprendre que la globalisation des échanges pourrait bien ne correspondre en rien à cette uniformisation des cultures, tant redoutée par ailleurs. Les cultures ne s'unifient pas, elles se diversifient : dans les newsgroups d'Internet, on trouve déjà plus d'une centaine de forums consacrés à des échanges entre individus et associations de cultures différentes.

Il y a plus encore : par sa nature propre, un tel modèle interdit toutes ces tentations de crispation sur les vestiges d'un passé glorieux, que sont les repliements identitaires, les fièvres patrimoniales, et les scléroses des traditions hissées au rang de programmes communs d'une modernité désenchantée.

\section{La fin de l'idéologie patrimoniale ?}

C'est pourquoi il paraît nécessaire de proposer -à la lumière d'un tel modèle- une approche renouvelée des patrimoines (matériels et immatériels) et de leur nécessaire médiation ${ }^{1}$. La démarche adoptée vise en effet à privilégier, grâce aux réseaux, ces objets culturels non durables qui échappent par définition à la fixation muséale, car ils n'existent que pensés selon les catégories de la relation et de l'échange. Périssables, fragiles, volatiles, ces créations croisées tiennent tout entières dans les interstices et les plis d'une microphysique de la culture. C'est la raison pour laquelle l'idéologie patrimoniale ne leur convient guère. Or cette dernière domine une fin de siècle corsetée dans les rituels pauvres d'innombrables conservations et commémorations.

Est-ce en raison des étroites connivences de ces dernières avec l'économie marchande qui précipite monuments historiques, savoirfaire et outils délaissés dans les vides d'une industrie touristique stigmatisée par Adorno et Marcuse ? "Le patrimoine ethnologique et culturel prend la valeur d'une «matière première» à exploiter, un

1 Sur la thématique de la médiation des savoirs, cf. Recherches en Communication, $\mathrm{n}^{\circ}$ 4, Louvain-La-Neuve, 1995. 
capital symbolique à travailler sans relâche" s'inquiète MarieFrançoise Lanfant ${ }^{1}$.

Comment ne pas s'interroger en effet sur les excès et les doutes d'une société en voie de panthéonisation, commémorant à outrance un passé idéalisé parce que mort? Sommes-nous victimes de ce mal que Karl Popper appelait joliment "l'ascendant de Platon" cette nostalgie languide de l'immuable, imputant le changement à l'inévitable corruption?

Les musées fleurissent là où des modes de vie (agricoles ou urbains), des représentations du monde ont fait naufrage. Puisqu'on ne conserve que ce qui est hors de l'usage, le patrimoine se tient d'emblée du côté de la trace, du vestige. Par un étrange paradoxe, notre temps célèbre à grands frais des réalités qu'il a évacuées par ailleurs (usines désaffectées, hangars vides, activités agricoles disparues...) et remplacées par des musées industriels, celui de la mine à Lewarde, de la sidérurgie au Creusot, du textile à Fourmies-Trélon, sans oublier ces éco-musées fantômes où gît la mémoire d'un monde rural folklorisé.

Sans doute cette muséologie effrénée est-elle vouée à conjurer le péril qui menace toute civilisation: son inéluctable disparition, loi non écrite des créations humaines ; mais la conservation ne revientelle pas "à une autre mort, par embaumement et consommation touristique" ??

L'histoire de la muséographie est marquée par la volonté de soustraire les ouvres du passé à l'outrage du temps, louable mais tardif souci qui naquît au milieu du XVIII' siècle. Elle puisera ensuite dans le combat contre le "vandalisme" révolutionnaire qui menaçait châteaux, abbayes et sépultures royales, une durable raison d'être ${ }^{3}$ avant que la modernité trépidante, oublieuse des antiques panaches réveillés par la magie évocatrice des livres de pierre, ne mobilise toute son attention. Contemporaine de la naissance du romantisme, la patrimonialisation véhicule un imaginaire puissant actualisé par la pédagogie du souvenir.

D'ores et déjà, les festivités annoncées pour accueillir dignement l'an 2000 nous acclimatent à cette idée curieuse : la commémoration de l'avenir; tant il est vrai que le décompte des jours qui nous séparent de la date fatidique est un moyen habile de la rattacher à

1 M.-F. LANFANT, "Identité, Mémoire, Patrimoine et «Touristification» de nos sociétés", Sociétés, Paris, Dunod, nº 46, 1994, p. 436.

2 M. SERRES, op. cit., p. 178.

3 Cf. D. Poulot, Musée, nation, patrimoine : 1789-1815, Paris, Gallimard, 1997. 
notre présent, de l'apprivoiser dans la prose des jours lentement égrénés.

\section{Culture réticulaire et matrimoine}

A cette "logique des propriétaires" que l'idéologie patrimoniale commande, il est permis de substituer la proposition d'un désaisissement actif, marquée par l'ouverture et la transversalité. Toutefois quand on l'interroge, cette notion de patrimoine s'avère indissociable d'une logique de l'héritage, ancré dans les racines d'un sol et dans les murs de la maison du père.

Or qu'est-ce encore que la Méditerranée ? Moins un patrimoine, semble-t-il -c'est-à-dire une séparation, une clôture, un lignage revendiqué qui appelle la conservation à l'identique- qu'un matrimoine, ou si l'on préfère, une virtualité matricielle unifiante et non uniformisante, favorisant les navigations et les flux, une sorte de creuset actif au sein duquel s'élaborent les conditions de possibilité d'un monde vivant et prismatique.

Matrimoniale en effet la pensée qui précède la découverte de l'archéologie, lisible dans l'intrication des vestiges des civilisations qui se succèdent ; vivant palimpseste dont la ville de Byblos, dans l'actuel Liban, offre un troublant panorama choisi parmi de nombreux exemples possibles. Vingt et un niveaux d'occupations y ont été dénombrés (le relevé s'arrête volontairement à l'empire Ottoman). Les fouilles révèlent l'incroyable : pêle-mêle les huttes préhistoriques, les temples amorites, les monuments égyptiens, le théâtre romain, les colonnes et les chapiteaux hellénistiques. Chaque époque pratiquant le réemploi systématique et naturel des matériaux précédents, la Méditerranée se dresse toute entière dans ces superpositions et ces emprunts naïfs mais déterminés, comme si faire l'Histoire et faire de l'Histoire s'arc-boutaient sur deux positions inconciliables.

Cette positivité de l'oubli lève l'hypothèque sur l'action, et pondère le devoir de mémoire par le devoir d'oubli. Nietzsche est le premier à s'alarmer, dès 1874 , du danger de l'excès d'Histoire et de références au passé, demandant instamment de libérer le présent du passé. "Ne devons jamais (nous) être que des descendants" que des "héritiers" s'interroge-t-il ? Malade de la "fièvre historique", la modernité nourrit pour le passé un respect excessif qui la fige dans la passivité. "On assiste alors au spectacle répugnant d'une aveugle soif 
de collection, d'une accumulation infatigable de tous les vestiges d'autrefois... manie de l'antiquaille, jusqu'à une insatiable curiosité aussi vaine que mesquine" ${ }^{1}$. Les Grecs avaient conjuré ce péril par le truchement d'une culture ouverte et chaotique : "un chaos de formes et de conceptions exotiques, sémitiques, babyloniennes, lydiennes et égyptiennes, et leur religion une véritable guerre des dieux de tout l'Orient"2. Ils apprirent à organiser ce désordre selon leur génie propre, échappant ainsi à la répétition et à l'imitation qui transforment une culture en "décoration de la vie"3. Nietzsche célèbre par ces mots la vertu du modèle méditerranéen, dynamique de recombinaison et de reconfiguration infinies des dispositifs culturels.

En cette fin du XX $\mathrm{XX}^{\mathrm{e}}$ siècle, les réseaux électroniques nous offrent une alternative non négligeable : par leur plasticité, ils supportent sans crainte le poids de la totalité de la mémoire humaine. Banques de données, musées virtuels, bibliothèques d'images et de sons... nos appétits archivistiques ne sont guère menacés par les nouvelles technologies, au contraire. Celles-ci n'ignorent rien des attraits de la sauvegarde, mais elles la plient aux nécessités d'échanges singularisés, foisonnants, plus rapides, parcourant des distances plus grandes. Les réseaux n'abolissent pas les réalités qu'ils numérisent : elles continuent d'exister dans le cadre de leurs propres registres spatiaux et temporels. Mais de même que la presse en ligne n'est pas la presse classique affublée de moyens électroniques, la culture réticulaire n'est plus la culture traditionnelle dotée de performances techniques sophistiquées. C'est pourquoi le sens et la valeur d'une culture ne se tiendront jamais du côté des outils, mais auprès des usages spontanés, irrévérencieux et surtout éphémères qui s'originent en eux, en vertu du principe selon lequel "il n'y a d'existant que ce qui nous échappe, irréductiblement"4.

Au-delà de sa contribution à l'intelligibilité des nouveaux modes de production et des nouvelles aires de distribution des cultures, le modèle méditerranéen illustre bien cette pédagogie de l'oubli qui rend sa chance à l'avenir, en désignant la question centrale : non plus celle de savoir "Que dois-je garder ?" mais celle-ci : "Que suis-je en droit d'oublier?".

\footnotetext{
1 F. NIETzSCHE, Seconde considération intempestive. De l'utilité et de l'inconvénient des études historiques pour la vie, Paris, Flammarion, 1988, p. 99.

2 Ibid., p. 178.

3 Ibid., p. 179.

4 M. DE CERTEAU, La culture au pluriel, Paris, Éd. du Seuil, 1993, p. 213.
} 
Rappelons ici pour mémoire, cette "rupture qui, d'un coup, au temps des Lumières, retranchera le legs méditerranéen des mouvements du monde pour le tenir captif dans le musée et dans un système d'éducation" : c'est Georges Duby qui s'exprime ainsi, ne craignant pas d'ajouter que notre époque refuse "cette Méditerranée truquée"1.

C'est pourquoi, en conclusion, il convient de souligner le bienfondé de l'application de ce modèle aux défis des temps qui viennent : dès lors plutôt que de redouter par trop le devenir incertain des cultures, proposons, grâce aux nouvelles technologies, de méditerranéiser les cultures à venir, au sens défini dans cet article. C'est ainsi que se peut concevoir, sans frayeurs inutiles ni invalidantes, la poursuite de l'aventure méditerranéenne ${ }^{2}$.

1 G. Duby, "L'héritage", in F. Braudel, G. Duby, La Méditerranée, tome 2, Les hommes et l'héritage, Paris, Flammarion, 1986, p. 214.

2 Cet article est une version étendue et remaniée d'une communication présentée par l'auteur au Colloque Européen "Méditerranée, Identités et Médiation du Patrimoine" qui s'est tenu au Musée Matisse à Nice, les 6, 7 et 8 mars 1997. 